\title{
Laboreal
}

Volume $16 \mathrm{~N}^{\circ} 2$ | 2020

Programa de Pesquisa do Curso da Ação

\section{Modelação sintética quantitativa de vários anos de atividade: qual é a relação entre significado na situação e complexidade longitudinal?}

Modelización sintética cuantitativa de varios años de actividad: ¿cuál es la relación entre la significación en situación y la complejidad longitudinal? Modélisation synthétique quantitative de plusieurs années d'activité: quelle articulation entre signification en situation et complexité longitudinale? Quantitative synthetic modelling of several years of activity: hat is the relationship between meaning in situation and longitudinal complexity?

Julien Guibourdenche

\section{OpenEdition}

Journals

Edición electrónica

URL: http://journals.openedition.org/laboreal/16776

DOI: $10.4000 /$ laboreal. 16776

ISSN: 1646-5237

Editor

Universidade do Porto

Referencia electrónica

Julien Guibourdenche, « Modelação sintética quantitativa de vários anos de atividade: qual é a relação entre significado na situação e complexidade longitudinal? », Laboreal [En línea], Volume 16 No2 | 2020, Publicado el 01 diciembre 2020, consultado el 13 diciembre 2020. URL : http:// journals.openedition.org/laboreal/16776; DOI : https://doi.org/10.4000/laboreal.16776

Este documento fue generado automáticamente el 13 diciembre 2020.

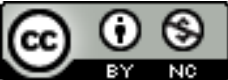

Laboreal está licenciado com uma Licença Creative Commons - Atribuição-NãoComercial 4.0 Internacional. 


\section{Modelação sintética quantitativa de vários anos de atividade: qual é a relação entre significado na situação e complexidade longitudinal?}

Modelización sintética cuantitativa de varios años de actividad: ¿cuál es la relación entre la significación en situación y la complejidad longitudinal? Modélisation synthétique quantitative de plusieurs années d'activité: quelle articulation entre signification en situation et complexité longitudinale? Quantitative synthetic modelling of several years of activity: hat is the relationship between meaning in situation and longitudinal complexity? Julien Guibourdenche

\section{NOTA DEL EDITOR}

Manuscrito recibido en: 02/03/2020

Aceptado tras peritaje en: 26/10/2020

Artigo publicado em língua francesa na revista Activités, vol. 17, no2 de 2020 [Le programme de recherche cours d'action]

Traducción : Agustina Blanco [agustinablanco@gmail.com]

El autor desea agradecer a los participantes en este estudio, así como a todos los actores del proyecto Smart Electric Lyon, especialmente a Pascal Salembier, Germain Poizat, Mariane Galbat e Yvon Haradji. El proyecto Smart Electric Lyon fue apoyado por la ADEME (agencia francesa de medioambiente y control de energía, por sus siglas en francés), en el marco del programa Investissements d'Avenir. El autor es el único responsable de las declaraciones vertidas en el presente artículo y declara no poseer ningún conflicto de intereses. 


\section{Introducción}

1 A pesar del interés de la ergonomía por las dinámicas temporales, numerosos son los desafíos que aún requieren una mejor comprensión de los tiempos largos y temporalidades múltiples de la actividad humana: la salud y las enfermedades profesionales (Mardon, Buchmann, \& Volkoff, 2013), la gestión temporal (Zara-Meylan, 2013, 2016), el desarrollo de prácticas profesionales (Chizallet, Prost, \& Barcellini, 2019), la sustentabilidad (Fréjus, 2019; Thatcher, Guibourdenche, \& Cahour, 2019). El problema general radica en que comprender la actividad significa situarla en el "aquí y ahora" de su aparición, teniendo en cuenta a su vez que esta última es "estructurada por y estructurante de" temporalidades lejanas y plurales. Los trabajos sobre los cursos de vida de agricultores (Barbier, Cerf, \& Lusson, 2015) o sobre la diacronía de actividades narrativas (Bationo-Tillon, Folcher, \& Rabardel, 2010) lo ejemplifican. Daniellou (2005), por su parte, habla de la actividad como un trozo de vida ( $a$ slice of life). Este aspecto es recurrente en los estudios longitudinales de la actividad y provoca el surgimiento de importantes retos para la investigación en materia de ergonomía. Más allá de los meollos conceptuales, a menudo resulta imposible analizar con fineza todos los datos (verbatims, videos, fotos, estadísticas, etc.) a lo largo de varios años. Se puede pasar entonces de niveles analíticos locales a niveles generales, sin una auténtica teoría explícita de la complejidad longitudinal de la actividad estudiada. Esto no atenta en absoluto contra el interés de los estudios actuales, pero una teoría general acerca de las dinámicas longitudinales de la actividad permitiría mejorar o facilitar los trabajos futuros. A la inversa, a veces es tentador aplicar marcos predefinidos (p. ej., Harbich \& Hassenzahl, 2017) a vastas extensiones temporales para abarcarlas de modo más completo. Pero la interpretación de los datos en relación con la actividad construida por los actores plantea interrogantes delicadas. ¿Cómo asegurarse de su validez desde el punto de vista del actor en situación de trabajo?

2 Esos retos reanudan aquí la pertinencia de la reflexión de Theureau (2006) sobre la articulación entre modelo analítico y modelo sintético en el programa de investigación Curso de Acción ( $\mathrm{CdA}$ ) [Curso de vida]. En el cierre de la obra Cours d'action - Méthode développée (Curso de acción - Método desarrollado), Theureau (2006) introduce varias categorías distintas de métodos y modelos (analítico o sintético, empírico o práctico, etc.). Esto se inscribe en la continuidad de sus reflexiones sobre el concepto de modelo (Badiou, 1969), sobre los modelos de análisis de las situaciones de trabajo complejas (véase Amalberti, Montmollin, \& Theureau, 1991), así como sobre los modelos y la complejidad desde una perspectiva enactiva (Theureau, 2005).

Un modelo empírico analítico concretiza las categorías descriptivas del campo de estudio y contribuye así a revelar características específicas de la actividad (Theureau, 2006). Este tipo de modelo está sumamente difundido tanto en ergonomía como en análisis de actividad en general. El objeto teórico curso de vida relativo a una práctica ha dado lugar a diferentes modelos empíricos de evolución de la actividad en un tiempo largo (p. ej., Barbier et al., 2015; Haué, 2003). Por lo general derivado de un método de análisis cualitativo, un modelo empírico analítico es descriptivo y a veces gráfico. También se abre a propuestas prácticas de transformación para un campo de estudio/ intervención particular. Pero su construcción está limitada por el campo y por el tiempo de análisis cualitativo de los datos, más aún cuando se trata de trabajar durante temporalidades de varios años que conllevan una profusión de datos. 
Un modelo empírico sintético es menos preciso sobre el contenido de la actividad de un actor particular, ya que deriva de una aplicación sistemática de categorías genéricas preexistentes a un conjunto de datos (las categorías conocidas como "de codificación", para emplear un término corriente). El arquetipo del modelo sintético es matemático: está constituido por un conjunto de hipótesis que se presume completo en relación al campo de estudio, coherentes y abiertas a razonamientos deductivos (Theureau, 2006). Generalmente, suele ir acompañado de "montajes materiales" (grafos, diagramas, etc.) que lo representan o lo imitan. Más "genérico", el modelo sintético permitiría dar cuenta de manera más acabada y homogénea la producción de un fenómeno a lo largo de un espacio-tiempo extenso o de varios años. Ahora bien, si se lo utiliza solo, corremos el riesgo de interpretar de modo falaz el contenido específico de la actividad y de la experiencia vivida por el actor en esa actividad (Theureau, 2006): sus preocupaciones, su sentir, su interpretación de la situación, sus proyecciones y expectativas...

5 Lo ideal sería entonces articular esos dos tipos de modelos, de forma tal que se puedan desarrollar conocimientos anclados en las situaciones de los actores (análisis cualitativo), al igual que en las dinámicas a largo plazo (abiertas a una caracterización sintética matemática). Las categorías de los modelos analíticos pueden y deben servir para construir "modelos sintéticos más pertinentes que aquellos que serían construidos por medio de la mera intuición del modelizador" (Theureau, 2006, p. 344, traducción libre). ¿Pero cómo proceder y qué se puede esperar concretamente de tal articulación? En el marco de una investigación longitudinal sobre la apropiación, el presente artículo discute las condiciones de producción y la utilidad de semejante relación. Luego de posicionar nuestro trabajo entre los enfoques de la actividad y los enfoques de la complejidad temporal en ergonomía (sección 2), el artículo expone en la sección 3 nuestro marco de modelización analítico-sintética, desarrollada inicialmente en el proyecto Smart Electric Lyon. La sección 4 muestra que el paso a una modelización sintética cuantitativa válida se juega ante todo en las labores de recopilación, procesamiento de datos y encuadre de los análisis de los resultados. Los resultados de la sección 5 demuestran que la articulación propuesta permite sintetizar varios años de actividad, siendo capaces de especificar la significación de las situaciones locales que conducen a dinámicas de adopción o rechazo de los sistemas, así como de proporcionar nuevas hipótesis que nutran un razonamiento deductivo. Sobre esas bases, la discusión de la sección 6 abre perspectivas para continuar el desarrollo de aproximaciones a la complejidad temporal, entre una fenomenología de la actividad y/o de la experiencia vivida y las ciencias de la complejidad.

\section{2. ¿Qué modelos de complejidad longitudinal aplicar a la actividad individual?}

Las interrogantes relativas a la modelización longitudinal de la actividad plasman diferentes posicionamientos conceptuales y metodológicos en materia de ergonomía y human factors, con respecto al tiempo y a la actividad humana. Interesándose por los modelos de las ciencias de la complejidad y a su vez por los human factors (matemáticos, sintéticos, abstractos, cronológicos), nuestra articulación analítico-sintética se inscribe en primer lugar dentro de la tradición del análisis de actividad. 


\subsection{La complejidad longitudinal: ¿qué nexo establece con las ciencias de la complejidad?}

7 Las ciencias de la complejidad y sus modelos sintéticos ¿pueden suministrarnos una parte de las herramientas pertinentes para comprender de un modo distinto las dinámicas longitudinales de la actividad? En ciencias de los sistemas complejos, Boeing (2016) deja suponer que la aproximación visual a los modelos matemáticos de sistemas dinámicos no lineales permitirían detectar estructuras "ocultas" en los conjuntos de datos temporales complejos. Pero ¿podemos asimilar la actividad individual a un sistema dinámico no lineal, por ejemplo? Desde la perspectiva de los human factors, Guastello (2017) sostiene que la teoría de los sistemas dinámicos no lineales permitiría reformular los problemas de ergonomía que guardan relación con la evolución temporal de los sistemas complejos: los accidentes laborales, la resiliencia de un sistema, la carga cognitiva, los grados de libertad en el movimiento y también la coordinación de un equipo. Este último autor y Walker et al. (2010) abogan por una orientación aún más asumida de la ergonomía hacia las ciencias de la complejidad, a fin de contemplar de manera distinta los problemas que se le plantean. Asimismo, Thatcher (2016) parece ir en esa dirección al movilizar los modelos del Network Analysis (una forma de análisis de los sistemas complejos) para aprehender las dinámicas de transformación temporal del sistema de sistemas duraderos. Tales convocatorias se inscriben en un movimiento más vasto de evolución hacia las ciencias de la complejidad, incluyendo las ciencias de la educación (p. ej., Hilpert \& Marchand, 2018) y la psicología social dinámica (p. ej., Wiese, Vallacher, \& Strawinska, 2010).

El conocimiento de la dinámica longitudinal de la actividad humana en el sentido de una construcción situada, cultivada e historizada de la práctica, técnica y significación en acción, no forma parte de los ámbitos de aplicación citados o priorizados por estos actores. Simplemente no es el enfoque seleccionado. Por consiguiente, antes de aplicar tal o cual modelo matemático de complejidad para sintetizar una dinámica longitudinal de la actividad, es conveniente interrogarse acerca de la articulación analítico-sintética: sus fundamentos conceptuales, metodológicos, etc.

Hilpert y Marchand (2018) proponen una categorización interesante de los modelos o "montajes materiales" (Theureau, 2006) posibles en lo que atañe a los sistemas complejos. Los grafos de series temporales parecen pertinentes para la descripción temporal del comportamiento de un elemento particular de un sistema complejo (p. ej. un individuo dentro de un sistema de producción). Los grafos del análisis de redes y de la modelización dinámica son adecuados para niveles de organización diferentes que superan nuestro estudio. El desarrollo del presente artículo inicia la reflexión relacionando categorías analíticas de actividad con una síntesis cuantitativa, pasando por una serie temporal. ¿Qué es lo que permite tal articulación? ¿Qué esconde y qué implica frente a la evolución de la actividad humana entre situación y tiempo largo?

\subsection{Complejidad longitudinal en ergonomía francófona centrada en la actividad}

10 La ergonomía de lengua francesa presenta enfoques muy diversos de las dinámicas longitudinales de la actividad y los sistemas complejos. Es necesario señalar una evolución general del estado de los modelos, en el contexto de este artículo. Modelizar 
significa también reducir, describir, explicar o simular (Amalberti et al., 1991). Pero las últimas décadas resaltaron asimismo la importancia de la modelización en tanto acción dentro de los contextos de intervención cada vez más participativos y reflexivos: para la mediación de los conocimientos (con uno mismo, con el otro), para superar la contingencia del conocimiento de la situación y para co-construir perspectivas sobre bases empíricas firmes pero "puestas a prueba" (Albaladejo, Magda, Geslin, \& Salembier, 2009). En materia de "modelos longitudinales" de la actividad hallamos un panel muy amplio, que va desde descripciones muy locales por medio del análisis de verbatims hasta simulaciones multiagente de la evolución cronológica de tareas y efectos de la actividad. A continuación, describimos algunos de los enfoques y modelos más pertinentes para el presente artículo.

11 Las verbalizaciones y los verbatims de los actores pueden permitir caracterizar la complejidad longitudinal de la actividad. Zara-Meylan (2016) caracteriza la complejidad de las temporalidades largas y plurales que estructuran la actividad de horticultor, movilizando un enfoque radicalmente cualitativo y fenomenológico. Mediante el progresivo análisis abductivo de verbatims, la investigadora va develando confusiones temporales de marcos y las complejas configuraciones de la actividad de los actores. Esto le permite destacar varios tipos de temporalidades involucradas en esta actividad a largo plazo: tiempo impulsado por el pasado, tiempo atraído por el futuro, tiempo de apertura de lo posible... Nos parece que tales temporalidades hacen eco a las de actualidad, potencialidad y virtualidad dentro del enfoque semiológico del curso de acción (Theureau, 1992) inspirado en Peirce (Ver San Martin \& Poizat, en este número), o a aquellas de virtual, real y concebible en los trabajos de Chizallet et al. (2019). Esas nociones, entre otras, pueden ser caracterizadas por verbatims.

cronicas temporales, sumamente difundidas en ergonomía, no son evidentes, por más que sean útiles para la creación de un espacio de referencia común sobre las dinámicas longitudinales. A diferencia de las verbalizaciones, estas conllevan, en todo o en parte, una representación lineal homogénea que no aborda la vivencia del tiempo figurado (Zara-Meylan, 2016). Su principio mismo consiste en incluir el tiempo cronológico en la modelización, lo cual implica una reflexión de fondo sobre el estatus del cronos en relación con el tiempo vivido y con el tiempo de la actividad: kairós. Sería reductor, empero, limitar el tiempo representado por las "crónicas" al mero cronos, y nuestro estudio pretende articular estas dos formas de tiempo. La redacción en forma de crónicas puede incorporar fuertemente categorías de experiencia vivida y contenidos de actividad (p. ej., Barbier et al., 2015; Chizallet et al., 2019; Haué, 2003). Por otra parte, sus atributos sintéticos no son nada desdeñables a la hora de representar dinámicas de varios años en un espacio restringido (p. ej., menos de una página).

13 Por último, existen articulaciones de enfoques modelizadores de la actividad humana y de la complejidad de los sistemas (Amalberti et al., 1991; Theureau, 2005) que brindan otras perspectivas para la investigación empírica y tecnológica en ergonomía. En esa vertiente, Haradji et al. (2018) articulan modelos analíticos situados de la actividad humana y modelizaciones matemáticas digitales sobre la base de un sistema multiagente, desde una óptica tecnológica de simulación de consumos de electricidad a gran escala. En ergonomía cognitiva de las situaciones deportivas, Bourbousson (2013) propuso pistas que permiten trabajar sobre la actividad colectiva de forma coordinada entre antropología cognitiva y teorías de los sistemas dinámicos. Estas muestran que 
los conceptos, métodos y modelos de la complejidad pueden dar lugar a concepciones fructíferas cuando se apoyan en miradas donde la actividad humana se tiene en cuenta de forma situada. Con todo, hasta donde sabemos, carecemos de modelos matemáticos sintéticos de la dinámica longitudinal de la actividad individual: un conjunto de hipótesis que permitan entrar en un razonamiento deductivo a propósito del "devenir" de una actividad individual en un tiempo de varios años. Si la actividad está en un estado A en el instante $t$, ¿qué debemos esperar respecto del instante $t+n$, y por qué?

En ese contexto, si la construcción de modelos de la complejidad adecuados es un desafío mayúsculo de la intervención y la investigación en ergonomía (Amalberti et al., 1991; Wisner, 1972), ¿qué hay de la dinámica longitudinal de la actividad individualsocial? ¿Resulta posible articular un modelo de la significación de la acción con un enfoque más matemático de su evolución? ¿Con qué condiciones y para aportar qué?

\section{Un observatorio de la dinámica longitudinal de calidad de experiencia vivida}

El presente artículo es un "paso lateral" que surgió en el marco del proyecto Smart Electric Lyon (Guibourdenche et al., 2019), inspirándose en contribuciones provenientes del programa de investigación empírica y tecnológica Cours d'action (Theureau, 2005, 2006) y otros trabajos en el área de psicología ergonómica (Cahour, Salembier \& Zouinar, 2016) y de long-term user experience (Harbich \& Hassenzahl, 2017; Karapanos, Martens, \& Hassenzahl, 2012; Kujala, Väänänen-Vainio-Mattila, Karapanos, \& Sinnelä, 2011).

\subsection{Un estudio sobre la apropiación de los sistemas de gestión de energía en el ámbito residencial}

La cuestión de la complejidad longitudinal nos ha sido planteada a través del tema de la apropiación de los sistemas de gestión de energía residencial. La apropiación se aborda aquí de manera doble: como proceso de integración al mundo y a la cultura propio de un actor (Theureau, 2006) y como generador de adopción/rechazo de una técnica. Por ejemplo, ¿cómo aprende un actor a activar un modo de gestión económica de su vivienda? ¿Cómo incorpora el modo de funcionamiento del sistema a su propio conocimiento y modalidad de aprehensión del mundo? ¿En qué medida esa progresiva incorporación se abre hacia una adopción o un rechazo del sistema?

La apropiación de los sistemas de gestión de energía ofrece una situación de estudio interesante de la complejidad de las dinámicas temporales de la actividad humana. Esto se debe en parte a que el sistema de gestión permite que el actor determine y controle mejor su consumo a través del tiempo. Pero sopesar el impacto en el propio consumo de energía a menudo requiere disponer de datos que abarquen dos temporadas de calefacción (inviernos) o de consumo (años), con el fin de poder comparar dos meses similares de distintos años, o dos años diferentes, por ejemplo. ¿Qué sucede durante esos dos años para el actor? ¿A partir de qué expectativas iniciales? ¿Surgen nuevas expectativas después de las primeras utilizaciones? ¿A qué se asemeja el proceso de apropiación en función de esas expectativas? ¿Qué explica la adopción y el rechazo al cabo de varios años? 

lugar a una socialidad y una tecnicidad mucho más vasta a través del tiempo que si tratáramos únicamente los momentos de utilización del sistema técnico. Esos momentos pueden cobrar la forma de contactos y comunicaciones del actor (participante) con el proveedor de energía, con el constructor del sistema, con el instalador o el reparador, e inclusive con sus colegas, vecinos o amigos. ¿Cómo interactúan todos esos momentos entre sí con el paso del tiempo? ¿Revisten todos ellos la misma importancia desde el punto de vista de la actividad de apropiación del actor? ¿Cuáles son las zonas de confianza y de incertidumbre en la evolución temporal de tal trayectoria de apropiación? Aquí volvemos a plantear preguntas que en parte son comunes con los enfoques longitudinales en ergonomía, mencionados en la parte introductoria.

trabajar estas interrogantes, nos hemos basado en una articulación analíticosintética que abarca los distintos planos de una investigación en materia de ergonomía: conceptuales, metodológicos (recopilación y procesamiento), empíricos y tecnológicos.

\subsection{Un anclaje en el análisis cualitativo de la experiencia vivida de la actividad}

20 En los planos conceptuales, nos fundamos en la noción de experiencia vivida de la actividad, que estructuró globalmente nuestro observatorio longitudinal, luego en la hipótesis de una "dinámica de calidad de experiencia vivida". La experiencia vivida de una actividad está definida como el "oleaje de acciones, emociones, pensamientos y sensaciones perceptuales generadas durante la realización de una actividad, de las cuales el sujeto es o puede ser consciente" (Cahour et al., 2016, p.257, traducción libre). La experiencia vivida de la actividad en los momentos de apropiación de los sistemas de gestión de energía permite dar cuenta de las necesidades, limitaciones y expectativas de los actores que participan en el estudio, respetando el principio de la primacía de lo intrínseco (Theureau, 2005, 2006).

21 Entre 2014 y 2015, se abrió un período analítico de un año y medio (Figura 1) durante el cual se implementaron los siguientes principios: recopilaciones en situación por observación, verbalizaciones simultáneas y post hoc, entrevistas de puesta en situación, análisis cualitativo de datos. Ese período nos condujo (Figura 2, punto A) a la realización de diagnósticos analíticos sobre las situaciones, necesidades, limitaciones y expectativas de los participantes, así como hacia alternativas de diseño para el servicio y los sistemas técnicos. 
Figura 1: Observatorio longitudinal que combina métodos clásicos para el análisis cualitativo de una experiencia vivida (observaciones in situ, rastros de actividad apuntados en un cuaderno, cotejo de los rastros, entrevistas, puesta en situación) (ícones negros) con análisis cuantitativoscualitativos sintéticos (ícones biancas), que permiten documentar lo que luego hemos llamado calidad de la experiencia vivida. Se efectuaron 43 sesiones de recopilación con 13 participantes, de 2013 a 2016.

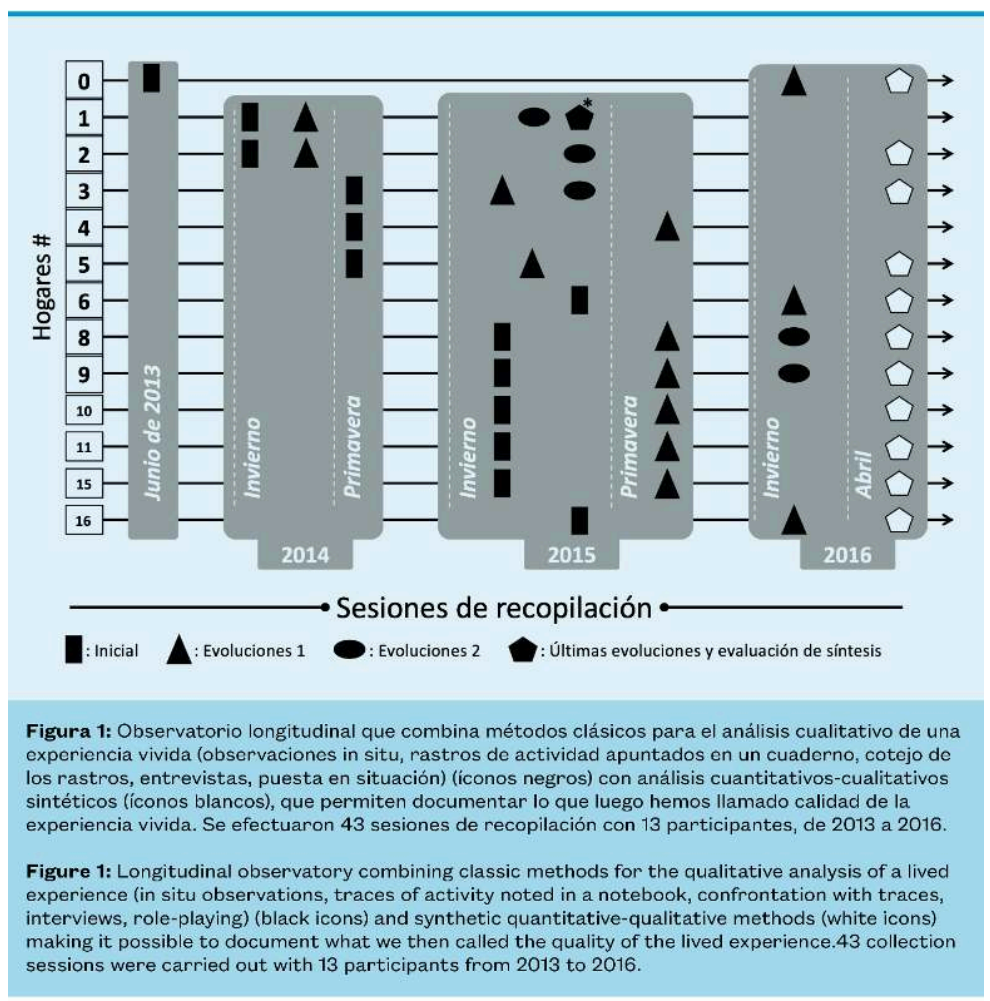

Figura 1: Observatorio longitudinal que combina métodos clásicos para el análisis cualitativo de una experiencia vivida (observaciones in situ, rastros de actividad apuntados en un cuaderno, cotejo de los rastros, entrevistas, puesta en situación) (ícones negros) con análisis cuantitativos-cualitativos sintéticos (ícones biancas), que permiten documentar lo que luego hemos llamado calidad de la experiencia vivida. Se efectuaron 43 sesiones de recopilación con 13 participantes, de 2013 a 2016

Este período analítico también asistió a la aparición de las primeras formalizaciones gráficas de las dinámicas de apropiación (no presentadas aquí), las cuales caracterizan las interacciones entre las expectativas y preocupaciones de los participantes y los acontecimientos significativos en los momentos de apropiación a lo largo del tiempo: un desperfecto, una baja de temperatura, un viaje por vacaciones... Una vez llegados al final de nuestros análisis de 2014 y 2015, se elaboran los primeros diagnósticos sobre la apropiación. Buscamos entonces desarrollar la investigación metodológica sobre esos aspectos longitudinales. Como punto de referencia, hasta el verano de 2015, el corpus de datos transcritos completamente (verbatims, fotos, observaciones) contiene alrededor de 600 páginas. En la discusión del informe de avance del proyecto, introducimos una primera representación sintética dimensional bajo la forma de radar. Además, indicamos que que ciertas dimensiones que caracterizan la calidad de la experiencia (facilidad, confort térmico) regresan de forma recurrente en los momentos de apropiación, para orientar positiva o negativamente las trayectorias hacia el rechazo o la adopción. Eso constituye el primer giro entre el fin del análisis cualitativo y el inicio del enfoque sintético de la dinámica de calidad de la experiencia vivida (Figura 1 pentágonos blancos y Figura 2 puntos B, C y D). 


\subsection{La calidad de la experiencia vivida: un objeto de estudio que conduce a una síntesis}

La noción de "calidad de la experiencia vivida" nos permite aquí tomar otra perspectiva, ya que habría sido imposible sistematizar un análisis de curso de vida que abarcara 13 hogares durante 3 años. Se optó por realizar una labor de análisis sistemática que luego sirviera de base para establecer una síntesis, según el movimiento propuesto por Theureau (2006). Al comienzo de este trabajo, la noción de "calidad de la experiencia vivida" no estaba sino implícita. La idea se aproxima a lo que podemos entender más usualmente por "experiencia del usuario". Entendemos por "calidad de la experiencia vivida" un conjunto de dimensiones cualitativas que tematizan la experiencia vivida pero que no le dan una forma intrínseca, dado que esa forma está reservada a la propia experiencia vivida, la conciencia prerreflexiva y el surgimiento de las significaciones en la acción (noción de informo para Theureau, 2006). Las aproximaciones en el área de UX research, psicología ergonómica o ergonomía proveen dimensiones que atañen a las emociones, el placer y el hedonismo de una forma complementaria a las "clásicas" dimensiones de utilidad y facilidad de utilización.

Figura 2: Evolución de nuestro método de estudio longitudinal de la actividad, dei análisis hacia la síntesis.

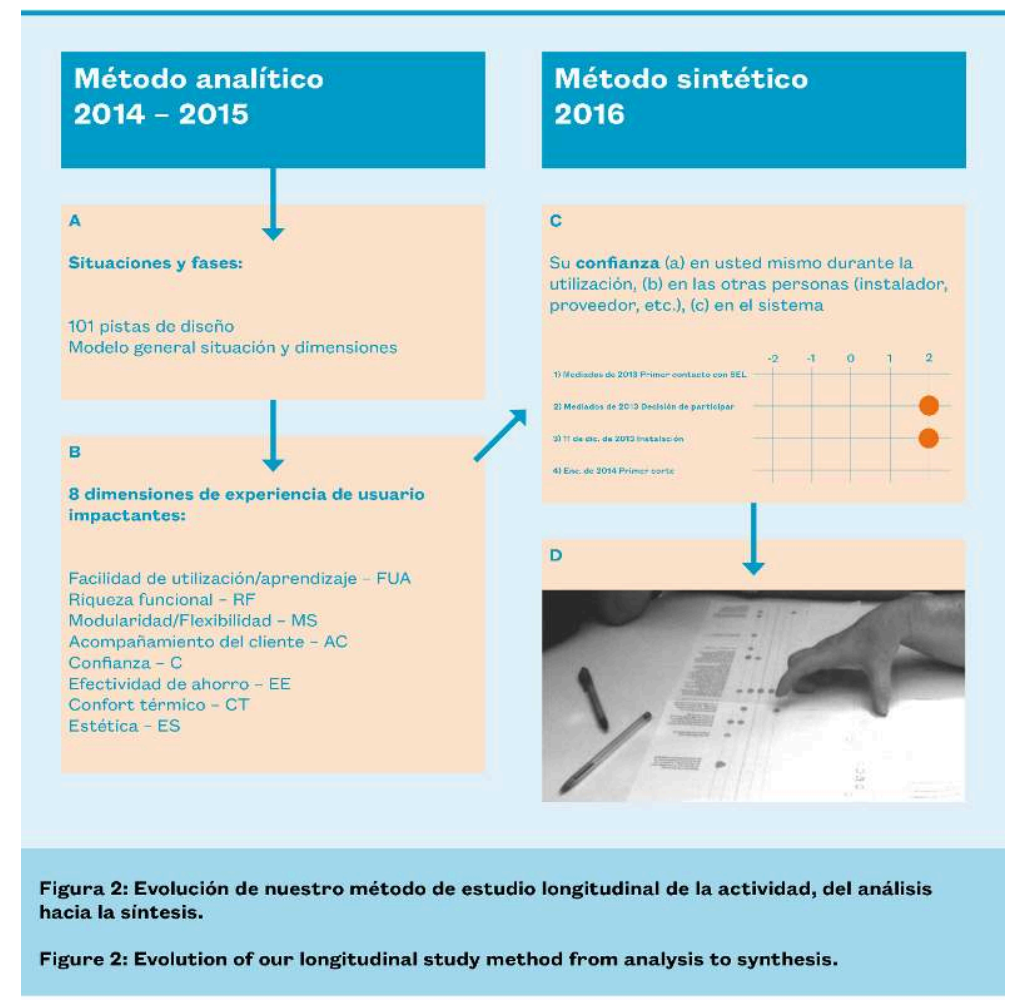

Figura 2: Evolución de nuestro método de estudio longitudinal de la actividad, dei análisis hacia la síntesis.

No hemos retomado tales dimensiones directamente, pues la calidad de la experiencia vivida puede ser caracterizada por un análisis y síntesis "inventivos" [2] (Theureau, 2005). Nuestra síntesis se funda en 8 dimensiones provenientes del trabajo de terreno (extraídas de nuestro corpus de 600 páginas, Figura 2 punto B), en un desarrollo 
metodológico (puntos C y D) libremente inspirado en las curvas UX e iScale (Karapanos et al., 2012; Kujala et al., 2011) y en los principios de las entrevistas destinadas a cotejar las huellas de la actividad (Cahour et al., 2016; Theureau, 2010). Sobre la base del corpus de las 28 recopilaciones efectuadas entre 2013 y 2015, el desarrollo se realizó colectivamente y de manera contradictoria en el equipo.

Figura 2, Punto A - Como hemos explicado en la sección anterior, los años 2014 y 2015 estuvieron dedicados a la realización de diagnósticos analíticos sobre las situaciones, necesidades, limitaciones y expectativas de los participantes, así como a la formalización de las alternativas de diseño para el servicio y los sistemas técnicos. En el verano de 2015, el trabajo de investigación sobre la caracterización de la calidad de la experiencia arranca con un nuevo procesamiento del corpus de 600 páginas constituido hasta entonces.

Figura 2, Punto B - Así, en febrero de 2016, se identificaron de modo consensuado en el equipo 8 dimensiones: la facilidad de utilización y de aprendizaje (FUA), la efectividad del ahorro (EE), el confort térmico (CT), el acompañamiento (AC), la confianza (C), la modularidad y la flexibilidad (MS), la riqueza funcional general (RF) y la estética (ES).

Figura 2, punto $\mathrm{C}$ - Con el objeto de dar cuerpo a la dinámica de "calidad de la experiencia vivida" a través del tiempo, desarrollamos una crónica denominada Chronovec (Figura 2, puntos C y D). Para cada verbalización:

a. identificamos y nombramos el momento (p. ej., contacto con el proyecto, instalación, viaje por vacaciones, etc.),

b. identificamos el tipo de dimensión en cuestión (una o varias entre las 8 antes citadas),

c. evaluamos la calidad de la experiencia vivida en cada dimensión significativa del momento, por medio de un puntaje sintético sobre una escala con 5 ítems (-2 (muy negativo), -1 (bastante negativo), 0 (neutro), +1 (bastante positivo), +2 (muy positivo). Por ejemplo, una experiencia vivida que implicaba una confianza en sí mismo muy negativa a raíz de un viaje por vacaciones valía - 2 en la dimensión Confianza en ese momento preciso,

d. elaboramos un diagnóstico sintético del momento (p. ej., "Poca confianza en sí mismo para utilizar el botón 'Ausencia' a raíz de un viaje por vacaciones").

28 Figura 2, punto D - Los investigadores llevaron a cabo una primera evaluación cuantitativa sintética previa a la última recopilación sintética, representada por el punto D. Durante la evaluación, la pauta era que esos momentos y esas dimensiones fueran evaluados por los mismos participantes, desde el punto de vista de su propia experiencia vivida y de su calidad (véase sección 4.1). Las notas entregadas por ellos prevalecían sobre las nuestras si existía una diferencia plausible (plausibilidad evaluada a través de los datos longitudinales disponibles y la última entrevista).

29 Al final de la entrevista de evaluación sintética, cada participante fue invitado a pronunciarse sobre su deseo de adopción o de abandono/rechazo del sistema (o sea, tras al menos dos temporadas de calefacción). Esas últimas verbalizaciones nos permitieron establecer un diagnóstico en cuanto al desenlace del proceso de apropiación en términos de adopción o abandono. Tal diagnóstico fue discutido de forma contradictoria en el equipo de ergónomos y comparado con la totalidad de los datos recabados, a fin de no remitirse únicamente a una evaluación del tiempo "t". Consideramos que había adopción si el/la participante deseaba conservar el sistema en su casa sin reservas y si la trayectoria de apropiación discutida en el equipo era 
consistente con esto. Por el contrario, cuando una persona emitía el deseo de adoptar el sistema pero con reservas y/o se topaba con dificultades de utilización después de varios años, estimamos que la adopción era real pero "parcial". Por último, los deseos de abandono del sistema fueron considerados como tales cuando resultaban coherentes con la trayectoria general en los diferentes años. La construcción del marco analíticosintético con 3 focos, presentado en las secciones 4.3 y 5 , fue útil para discutir en profundidad los aspectos coherentes y las sorpresas en las trayectorias.

Las siguientes secciones presentan los retos que supone pasar del punto D a la modelización sintética de la complejidad longitudinal de la calidad de la experiencia vivida. Nos basamos en un corpus final de 970 páginas, que caracterizan 39 momentos en promedio por hogar (de los 13 hogares iniciales, finalmente 10 fueron procesados después de abril de 2016). El promedio del largo de la trayectoria de apropiación descrita es de 1 año y 5 meses; 3 años como máximo y 11 meses y medio como mínimo. Los participantes entregaron 1.308 notas al momento de la evaluación de síntesis.

\section{Un trabajo de articulación de modelos se pone en juego desde la recopilación hasta el procesamiento}

31 Antes de visualizar y discutir los gráficos de series temporales, la transición hacia una modelización sintética cuantitativa válida se juega ante todo en el trabajo de recopilación, en la labor de procesamiento de datos y de encuadre del análisis de los resultados.

\subsection{Cuantificar asegurando un nexo con la vivencia: entre trazas y guía en la entrevista}

La actividad de evaluación sintética de la calidad de la experiencia nos muestra la gran importancia de un trabajo de confrontación a trazas y de puesta en situación en los casos que suponen varios meses/años de estudios. El uso de trazas de la actividad, la creación de soportes mnésicos y la puesta en situación nos parecieron centrales para una cuantificación realista de la dinámica temporal de la calidad de la experiencia vivida.

La Figura 3 presenta un fragmento de las verbalizaciones de la entrevista de evaluación de síntesis con la participante 16 en abril de 2016 (véase Figura 1: ícono blanco a la derecha, últimas evoluciones y evaluación de síntesis). La consigna inicial era evaluar la facilidad de utilización y de aprendizaje en los momentos presentados en la crónica y, a su vez, verbalizar la vivencia de ese momento en voz alta. En los momentos 18 y 24 (Figura 3), la participante 16 evalúa la facilidad de utilización y de aprendizaje en +2 , siendo que según nuestros registros y análisis precedentes no se sentía cómoda con el sistema. Se trata de una racionalización post hoc. Se imagina que para ella era fácil, pues ahora es más fácil en virtud de la apropiación parcial que tuvo lugar (sobre todo, a través del acompañamiento brindado). No intervenimos de inmediato respecto de los momentos 18 y 24 para no exponerla a una incoherencia. Lo cierto es que dice "ausencia larga, ahora siempre pongo ausencia larga (...) entonces así está muy bien", lo cual indica una falta de evocación de la experiencia vivida en situación. El momento 26 la conduce a un recuerdo relevante: su mail de solicitud de explicación sobre su sistema 
a propósito de sus ausencias cortas (“¡No me respondieron!”). Y a su vez, ese recuerdo desata una interpretación sobre la facilidad de utilización: "Así que antes no era tan fácil". La participante se da cuenta de que sus evaluaciones anteriores eran erróneas. Se coloca entonces en situación con respecto a su experiencia vivida en el momento 24 y reevalúa en consecuencia.

Figura 3: Fragmento de las verbalizaciones de la entrevista de evaluación de síntesis con la participante 16 en abril de 2016 (véase Figura 1: ícono blanco a la derecha). Se percata de que no le resultaba tan fácil utilizar el modo "Ausencia" antes de diciembre de 2015 y luego corrige las notas en consecuencia.

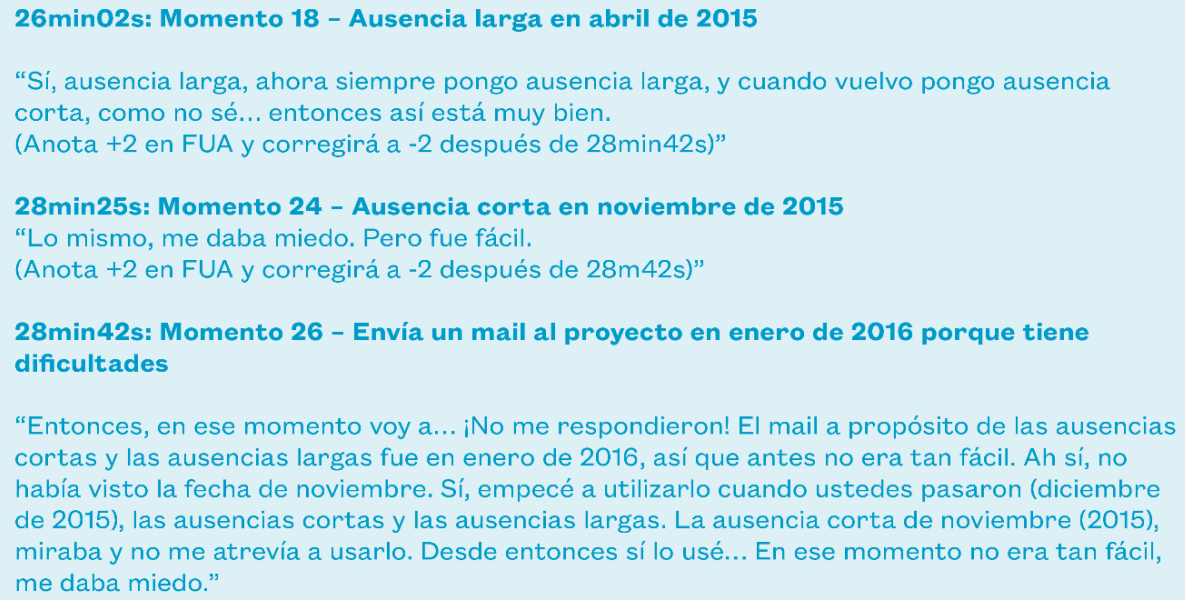

Figura 3: Fragmento de las verbalizaciones de la entrevista de evaluación de síntesis con la participante 16 en abril de 2016 (véase Figura 1: ícono blanco a la derecha). Se percata de que no le resultaba tan fácil utilizar el modo "Ausencia" antes de diciembre de 2015 y luego corrige las notas en consecuencia.

Figure 3: Excerpt from the synthetic evaluation interview verbalisations with participant 16 in April 2016 (see Figure 1: white icon on the right). She realizes that she did not find it as easy to use the absence mode until December 2015 and then corrects the scores accordingly.

Figura 3: Fragmento de las verbalizaciones de la entrevista de evaluación de síntesis con la participante 16 en abril de 2016 (véase Figura 1: ícono blanco a la derecha). Se percata de que no le resultaba tan fácil utilizar el modo "Ausencia" antes de diciembre de 2015 y luego corrige las notas en consecuencia.

Toda entrevista de puesta en situación engendra formas plurales de evocación de la experiencia vivida y verbalizaciones que de allí se desprenden. A veces las verbalizaciones son demasiado genéricas y no especifican nada de la experiencia en el momento considerado. A veces, como en el ejemplo anterior, se manifiesta una incoherencia evidente entre lo que se ha dicho en el pasado y lo que se dice en la evaluación de síntesis. Tales formas particulares de navegación entre pasado imaginado o real y entre posiciones de evocación situada o de reflexión son de esperar en el caso de comentarios que versan sobre meses y años de experiencia. En ese contexto, relevamos 6 exigencias para la cuantificación a partir de la recopilación de síntesis, las cuales se añaden a la necesidad general de guiar las entrevistas de puesta en situación (p.ej., explicitación, autoconfrontación):

1. Desde la introducción de la consigna, el/la participante debe ser conducido/a a verbalizar la experiencia vivida en el momento cuantificado, sea cual fuera la nota, 
2. El/la participante debe ser conducido/a a no cuantificar si el momento y la dimensión no le recuerdan nada significativo,

3. La crónica de los momentos (Figura 2, puntos C y D) debe ser presentada de modo que suministre un soporte de confrontación con la actividad,

4. Ese soporte debe ser presentado exento de las evaluaciones de los investigadores,

5. El/la participante puede completar los momentos que le son presentados en el transcurso de la evaluación con aquellos que se le ocurran durante la evaluación,

6. Es importante para el evaluador realizar algunos análisis previos a la evaluación de síntesis para poder volver a colocar al o a la participante en situación.

\subsection{El procesamiento de los datos cuantitativos: fecundidad y peligro de la "ceguera"}

Los datos de las evaluaciones de síntesis fueron transcritos en su integralidad. La labor de articulación analítico-sintética da origen luego (1) a la creación de un cuadro que pone en relación las notas con los verbatims y (2) a la creación de un marco para el procesamiento de datos cuantitativos (Figura 4), sumado a la definición de un marco analítico.

En referencia a la obra de Singer y Willett (2003) sobre el análisis de los datos longitudinales, nuestro cuadro de datos básicos (Figura 4) contiene para cada participante: (1) un nombre de usuario (una hoja de cálculo por participante), (2) un indicador de tiempo (momentos: del 1 al último) y (3) los valores de las notas entregadas para cada dimensión en cada momento. Además, quisimos conservar los datos brutos para poder acceder a diferentes focos de modelización y no sólo al promedio de los puntajes por momentos, por ejemplo. Por consiguiente, guardamos rastros de los puntajes negativos, neutros y positivos en cada momento. La hoja del cuadro básico (Figura 4) contiene 24 columnas, lo cual resulta del cruce de cada una de las 8 dimensiones con cada tipo de valencia posible: positiva, neutra y negativa. Si una dimensión no es significativa en relación con la experiencia vivida en el momento, entonces no hay nota; en aras de validez ecológica. 
Figura 4: Fragmento dei cuadro básico de un participante. Para cada momento, se transcriben los puntajes negativos, neutros y positivos. FUA facilidad de utilización y de aprendizaje EE efectividad dei ahorro $\mathrm{CT}$ confort térmico. AC acompanamiento $\mathrm{C}$ conftanza MS modularidad y flexibilidad RF riqueza funcional ES estética.

\begin{tabular}{|c|c|c|c|c|c|c|c|c|c|c|c|c|c|c|c|c|c|c|c|c|c|c|c|c|}
\hline \multirow{2}{*}{ Momentos } & \multicolumn{8}{|c|}{ Puntajes negativos } & \multicolumn{8}{|c|}{ Puntajes nuestros } & \multicolumn{8}{|c|}{ Puntajes positivos } \\
\hline & $A C$ & FUA & EE & MS & c & CT & $\mathrm{RF}$ & ES & $A C$ & FUA & EE & MS & c & CT & RF & ES & $\mathrm{AC}$ & FUA & EE & MS & c & CT & $\mathrm{RF}$ & ES \\
\hline 1 & & & & & & & & & & & & & & & & & & & 2,0 & & & & & \\
\hline 2 & $-2,0$ & & $-2,0$ & & $-2,0$ & & & $-2,0$ & & & & & & & & & & & & & & & & \\
\hline 3 & $-2,0$ & & $-1,0$ & & & & & & & & & & & & & & & & & & 1,0 & & & \\
\hline $3 b$ & & & & & $-2,0$ & & & & & & & & & & & & & & & & & & & \\
\hline 4 & $-1,0$ & & & & & & & & & & 0,0 & & & & & & & & & & & & & \\
\hline 5 & & & & & & & $-1,0$ & & & & $-2,0$ & & & & & & & 2,0 & & & & & 2,0 & \\
\hline \multicolumn{25}{|c|}{$\begin{array}{l}\text { Figura 4: Fragmento del cuadro básico de un participante. Para cada momento, se transcriben los puntajes negativos, } \\
\text { neutros y positivos. FUA facilidad de utilización y de aprendizaje EE efectividad del ahorro CT confort térmico } \\
\text { AC acompañamiento C confianza MS modularidad y flexibilidad RF riqueza funcional ES estética. }\end{array}$} \\
\hline $\begin{array}{l}\text { Figure 4: } E \\
\text { negative, } n \\
\text { CT thermal }\end{array}$ & $\begin{array}{l}\text { xcer } \\
\text { eutr } \\
\text { com }\end{array}$ & $\begin{array}{l}\text { pt fro } \\
\text { al and } \\
\text { fort A }\end{array}$ & $\begin{array}{l}\text { m ap } \\
\text { posi } \\
\text { a sup }\end{array}$ & $\begin{array}{l}\text { parti } \\
\text { itive } \\
\text { pport }\end{array}$ & $\begin{array}{l}\text { cipar } \\
\text { score } \\
\text { tC tr }\end{array}$ & $\begin{array}{l}\text { nt's s } \\
\text { es ar } \\
\text { ust, f }\end{array}$ & $\begin{array}{l}\text { prea } \\
\text { e tra } \\
\text { faith }\end{array}$ & $\begin{array}{l}\text { dshe } \\
\text { nscri } \\
\text { and c }\end{array}$ & $\begin{array}{l}\text { et. E } \\
\text { ibed. } \\
\text { onfid }\end{array}$ & $\begin{array}{l}\text { xcerp } \\
\text { FUA } \\
\text { lence I }\end{array}$ & $\begin{array}{l}\text { pt fro } \\
\text { ease } \\
\text { MS n }\end{array}$ & $\begin{array}{l}\text { om a } \\
\text { of us } \\
\text { modul }\end{array}$ & $\begin{array}{l}\text { part } \\
\text { e and } \\
\text { larity }\end{array}$ & $\begin{array}{l}\text { icipar } \\
\text { d learr } \\
\text { and } f\end{array}$ & $\begin{array}{l}n \text { nt's } \\
\text { ning } \\
\text { flexib }\end{array}$ & $\begin{array}{l}\text { basic } \\
\text { EE eff } \\
\text { ility R }\end{array}$ & $\begin{array}{l}\text { char } \\
\text { ffectiv } \\
\text { RF fun }\end{array}$ & $\begin{array}{l}\text { t. For } \\
\text { veness } \\
\text { nction }\end{array}$ & $\begin{array}{l}\text { reac } \\
\text { s of s } \\
\text { nal ric }\end{array}$ & $\begin{array}{l}\text { ch mor } \\
\text { savings } \\
\text { chness }\end{array}$ & $\begin{array}{l}\text { omen } \\
\text { sS ES }\end{array}$ & it, the & & \\
\hline
\end{tabular}

Figura 4: Fragmento dei cuadro básico de un participante. Para cada momento, se transcriben los puntajes negativos, neutros y positivos. FUA facilidad de utilización y de aprendizaje EE efectividad dei ahorro CT confort térmico. AC acompanamiento C conftanza MS modularidad y flexibilidad RF riqueza funcional ES estética.

Este momento y el momento de los primeros procesamientos y discusiones de series temporales conforman una "ceguera", en el sentido de que allí se juega a ruptura entre el análisis de la experiencia vivida y la síntesis cuantitativa ligada a las notas que evalúan la calidad de la experiencia vivida. Tal ruptura se produce debido a la actividad de procesamiento activo de los datos cuantitativos, lo cual conlleva un tiempo y una forma de pensar que difieren del análisis cualitativo inicial. En cierto sentido, esa ceguera es necesaria y potencialmente fecunda, ya que la ruptura permite confrontar más adelante los diagnósticos cualitativos y cuantitativos, analíticos y sintéticos; también permite dejarse sorprender por las formas de evolución visualizadas una vez que los datos han sido procesados correctamente. En otro sentido, es clásicamente peligroso, en la medida en que podría permitir todas las interpretaciones si no fuera luego ligada a la experiencia vivida gracias a los verbatims y los análisis cualitativos previos de 2014 y 2015.

\subsection{Un marco analítico-sintético con 3 focos}

Como devela la sección siguiente, finalmente desarrollamos un marco con 3 focos sobre la calidad de la experiencia vivida. Un foco es el agrupamiento de varios elementos de modelización: una escala temporal (un momento, varios momentos o la serie temporal de todos los momentos), un tipo de análisis (cualitativo o cuantitativo), una extensión de dimensiones (una dimensión en particular o todas) y un tipo de procesamiento cuantitativo (notas ajustadas en bruto, promedio o tendencia por regresión lineal).

Los distintos focos están numerados de 1 a 3 y caracterizan niveles progresivos de síntesis y abstracción. El foco cualitativo local $\left(\mathbf{n}^{\circ} \mathbf{1}\right)$ se construye a partir de las verbalizaciones de los actores sobre los momentos. Proporciona la base de los demás 
focos y nos permite efectuar diagnósticos cualitativos sobre la experiencia vivida en esos momentos y sobre la apropiación del sistema. El foco cuantitativo radial $\left(\mathbf{n}^{\circ} 2\right)$ se construye a partir del modelo radial con dos polos (Figura 7), que permite apreciar la valencia positiva/negativa de la experiencia según las dimensiones y su valor $(0,102)$. Ofrece acceso a la estructura de la calidad de la experiencia vivida cuantificada del momento, o a une serie temporal parcial de esos momentos. El foco cuantitativo serial $\left(n^{\circ} 3\right)$ se construye a partir de una o varias series temporales completas (Figuras 5 y 6 ) de notas ajustadas en bruto. Puede referirse a una sola dimensión (en ese caso, se lo llama dimensional), o a todas en cada momento (se lo llama general). Este foco va acompañado de la tendencia por regresión lineal en todos los momentos y aporta un esclarecimiento general en cuanto a la dinámica de calidad de la experiencia vivida a lo largo de varios años.

Una serie temporal representa la evolución de una sucesión finita de estados/ cantidades sobre un eje cronológico. Las series temporales son modelos estadísticos de complejidad mínima (Hilpert \& Marchand, 2018) que, en nuestro proyecto, permiten visualizar dinámicas longitudinales de otro modo. La construcción de nuestra "métrica razonable del tiempo" (Singer \& Willett, 2003) está basada en el punto de vista del actor y su propia construcción de significaciones durante los momentos de apropiación (por ejemplo, la instalación, el viaje por vacaciones, etc.). Por ende, si el intervalo temporal entre cada momento de apropiación es representado de forma homogénea para facilitar la lectura (p. ej. Figura 5), las duraciones cronológicas de los intervalos difieren, puesto que dependen de los momentos en los cuales tuvo lugar la experiencia. El intervalo puede ser un día, así como un mes. Según Singer y Willett (2003), esto cae bajo la órbita de un trabajo sobre los datos temporales llamados "no estructurados" (Singer \& Willett, 2003) [3]. Por lo demás, nuestras series temporales sólo son plenamente legítimas a partir del momento en que se abona la hipótesis de una continuidad de significaciones para el actor a través de un tiempo largo, tal como sucede, por ejemplo, con el objeto teórico curso de vida relativo a una práctica (Theureau, 2006). Por último, el objetivo asignado a esas series temporales es relativamente modesto: permitir tratar otros aspectos de la dinámica de apropiación que el análisis cualitativo no nos habría dejado ver.

\section{Utilidad de los focos y de su articulación}

\subsection{Foco 3 general: comparar las dinámicas longitudinales}

41 La primera utilidad del foco 3 general es la de examinar detalladamente las dinámicas de cada hogar a lo largo de toda la duración de la recopilación de datos. A pesar de sus evidentes límites ligados a un procesamiento en función del promedio general de los puntajes en cada momento, esto permite comparar visualmente las trayectorias entre sí, lo cual facilita una mirada con perspectiva respecto de los datos procesados. Por ejemplo, la Figura 5 muestra de manera patente que ciertas trayectorias denotan una calidad de vivencia globalmente positiva a través del tiempo (participante 11), allí donde otras arrojan diagnósticos más moderados (participante 16) o negativos (participante 15).

42 Combinado con los diagnósticos derivados del foco cualitativo local $\left(n^{\circ} 1\right)$, el foco 3 permite asimismo iniciar discusiones en cuanto a las relaciones entre la valencia de la 
experiencia (positiva o negativa) y la trayectoria de apropiación; o bien en cuanto al valor de cada momento en esa trayectoria. Por ejemplo, aquí vemos que todas las series temporales varían fuertemente entre valencias negativas y positivas a través del tiempo. Esto significa que todos los participantes vivieron varios momentos muy negativos y muy positivos. Por otra parte, observamos también que esto es cierto en el caso de una trayectoria que redunda tanto en una adopción como en un rechazo del sistema al final del proyecto (foco cualitativo local ( $\left.n^{\circ} 1\right)$ ). Por consiguiente, no existe relación simple y directa entre la valencia de la calidad de la experiencia vivida por el usuario y su adopción del sistema.

Tampoco existe relación simple entre la calificación negativa o positiva de la valencia y la incorporación del sistema. El foco cualitativo local $\left(n^{\circ} 1\right)$ nos mostró en varias oportunidades que determinadas experiencias vividas negativamente dan lugar a aprendizajes y a intercambios, favoreciendo la incorporación del sistema al mundo y a la cultura propios del usuario. En el momento de una avería o de una reparación, por ejemplo, los participantes pueden verse sorprendidos negativamente, pero también pueden recibir una buena atención por parte del servicio de asistencia. En esa ocasión, pueden aprender nuevas cosas (p. ej. cómo funciona una parte del sistema), que luego van a estructurar otros momentos de apropiación.

Figura 5: Agrupamiento de representaciones de las dinámicas longitudinales a partir de la serie temporal en el foco 3- general, con el objeto de facilitar la comparación entre los participantes. Los círculos negros corresponden ai puntaje promedio obtenido en todas las dimensiones significativas de un momento. La línea negra es la regresión lineal de los promedios de todas las dimensiones.

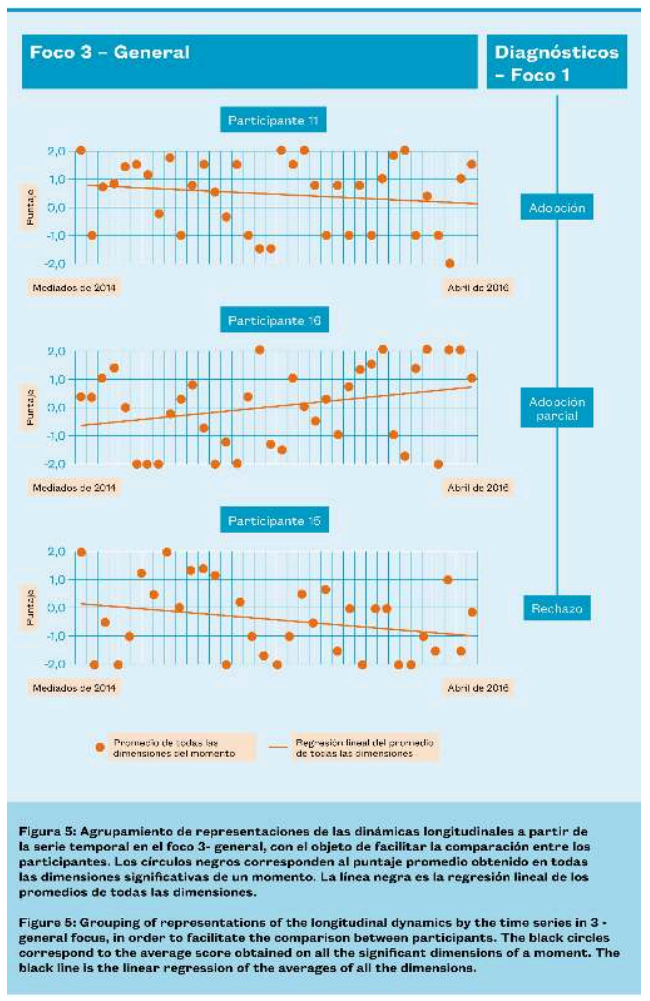

Figura 5: Agrupamiento de representaciones de las dinámicas longitudinales a partir de la serie temporal en el foco 3- general, con el objeto de facilitar la comparación entre los participantes. Los círculos negros corresponden ai puntaje promedio obtenido en todas las dimensiones significativas de un momento. La línea negra es la regresión lineal de los promedios de todas las dimensiones. 
cruces llevan a plantearse nuevas interrogantes. Respecto del participante 11 podemos preguntarnos cómo una dinámica general que tiende hacia un puntaje promedio más bien negativo o nulo puede corresponderse, con el correr del estudio, con una adopción. ¿Deberíamos anticipar un posible abandono en los años venideros? ¿Por qué motivos? ¿Habrá alguna dimensión que desempeñe un papel más importante que otras? La pregunta se invierte en relación con la participante 16: ¿cómo es posible partir de un polo tan negativo de experiencia y desembocar en una adopción parcial?

A partir de este tipo de constatación, resultan interesantes dos ópticas de procesamiento más avanzadas: uno que afine los aspectos estadísticos y el otro que esclarezca las articulaciones posibles entre el foco 3 cuantitativo serial y las significaciones construidas en situación (foco 1 cualitativo local), con miras a explicar esos puntajes en términos cualitativos. Presentamos esta segunda óptica en las siguientes dos subsecciones y regresaremos a la primera en la discusión final.

\subsection{Foco 3-dimensional: caracterizar los momentos y dimensiones claves}

Desde el punto de vista de la experiencia vivida, no todas las dimensiones ni todos los momentos están al mismo nivel. Algunos son "claves" y otros no. Desde la óptica del foco cualitativo local $\left(n^{\circ} 1\right)$, una dimensión clave remite a una preocupación/ expectativa muy fuerte del participante, de modo tal que prevalece sobre las otras. En el plano estadístico, una dimensión clave acentúa la tendencia del promedio general, siendo coherente a su vez con el diagnóstico cualitativo del foco cualitativo local $\left(\mathrm{n}^{\circ} 1\right)$. Para el participante 15, presentado a continuación en la Figura 6, la efectividad del ahorro (EE) representa una dimensión clave.

Laboreal, Volume 16 N² 2 | 2020 
Figura 6: Series temporales cuantitativas seriales general (círculos negros y recta continua) y dimensional respecto de la efectividad dei ahorro (EE - cuadrados grises y recta puntuada). En la abscisa: sucesión temporal de los momentos. En la ordenada: puntaje de la evaluación de la calidad de la experiencia vivida. Esta serie abarca 34 momentos, que van de mediados de 2014 a abril de 2016 para el participante 15.

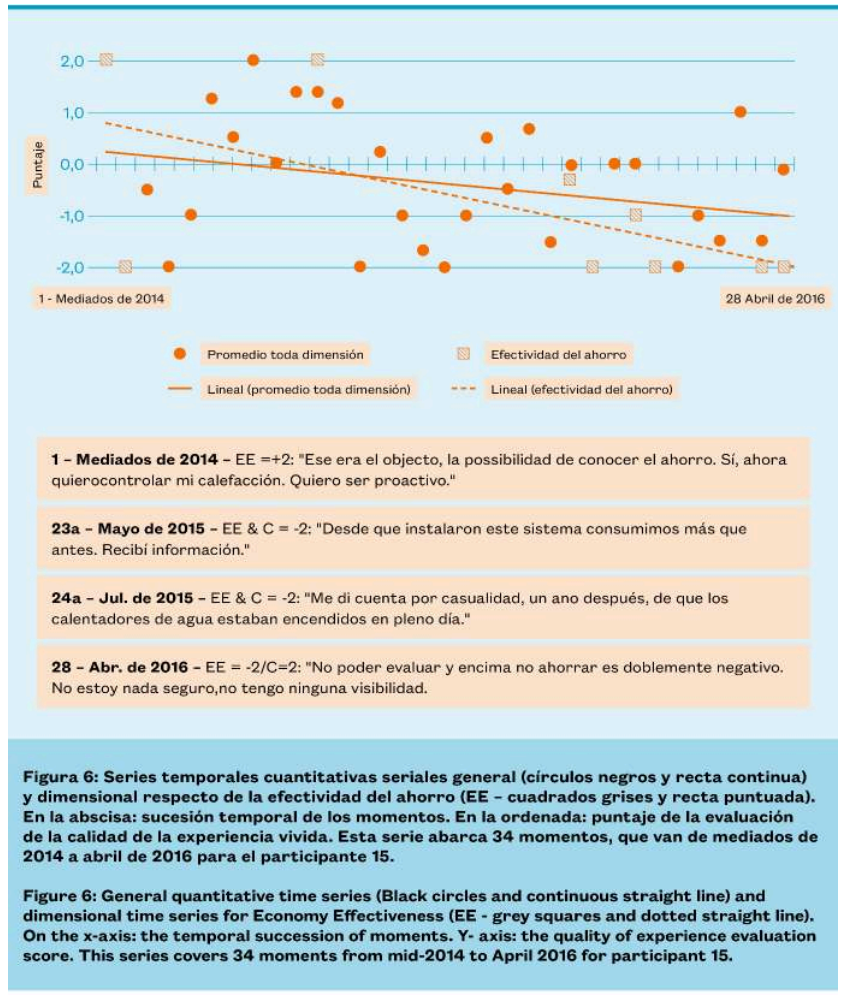

Figura 6: Series temporales cuantitativas seriales general (círculos negros y recta continua) y dimensional respecto de la efectividad dei ahorro (EE - cuadrados grises y recta puntuada). En la abscisa: sucesión temporal de los momentos. En la ordenada: puntaje de la evaluación de la calidad de la experiencia vivida. Esta serie abarca 34 momentos, que van de mediados de 2014 a abril de 2016 para el participante 15.

A nivel estadístico, en la Figura 6, la recta de regresión EE presenta un coeficiente más negativo que la recta del promedio del foco 3 general; el más negativo de todas las dimensiones, dicho sea de paso. Esto indica, pues, una dinámica de mayor amplitud desde el comienzo hasta el fin, en dirección al negativo. Más aún, con los verbatims del foco cualitativo local $\left(n^{\circ} 1\right)$, vemos hasta qué punto EE contaba para el participante 15; la dimensión EE es clave para él. En realidad, eso remite a un conjunto de abiertos/ posibles (Theureau, 2006) y de expectativas fuertemente estructurantes para la dinámica longitudinal de ese participante: conocer el ahorro, controlar mejor su calefacción, ser proactivo. Estas forman parte de su "mundo propio". En cuanto a la incorporación, vemos también que estos abiertos no entran en "colisión" con una experiencia crítica del sistema antes del mes de mayo de 2015 (momento 23a), es decir, casi un año después del comienzo de la trayectoria a mediados de 2014. De forma tal que durante alrededor de un año se viven experiencias positivas y negativas en torno al sistema, y la incorporación se va produciendo aunque no resulte en algo realmente decisivo con miras a la adopción. En el momento 23a, la integración del sistema a la experiencia vivida del participante se produce de forma muy negativa puesto que este comprueba un consumo excesivo, siendo que él esperaba conocer el ahorro y, sobre todo, controlar mejor su calefacción. Más adelante, el sistema se integra nuevamente de 
manera muy negativa (24a y 28 en particular) en la experiencia vivida, acarreando un rechazo al final de nuestra evaluación.

Este foco, siempre articulado con el foco cualitativo local $\left(\mathrm{n}^{\circ} 1\right)$, nos permitió desglosar el papel de cada dimensión de la experiencia en la dinámica de apropiación de cada participante. Por ejemplo, la efectividad del ahorro, cuando es clave, hace que el actor no se vea impactado de manera crítica en los primeros momentos de apropiación por las razones antes explicitadas. El efecto primero de una preocupación-expectativa clave ligada a EE es provocar que se sostenga el uso y las expectativas a largo plazo, antes de una o varias experiencias que sean verdaderamente decisivas. Por esto mismo, estamos autorizados a sintetizar la trayectoria comparando esa dimensión y los momentos claves que la ponen en juego en el plano de la experiencia vivida, si aquella presenta claramente esa dimensión como algo significativo. En cambio, no todos los hogares comparten las mismas preocupaciones y expectativas iniciales. En virtud de ello, la importancia de los primeros momentos de apropiación (p. ej. la familiarización) puede ser mayor para participantes que esperan, sobre todo, una facilidad de utilización (FUA) y/o que no tienen una fuerte preocupación en cuanto a la efectividad del ahorro. Así pues, algunos hogares participantes frenaron la utilización y descubrimiento del sistema durante la etapa de familiarización (inicio de trayectoria), al no entender cómo utilizarlo y al no tener ningún abierto/posible estructurante que habría favorecido la persistencia de alguna forma de apropiación y utilización.

Para terminar, el carácter clave de ciertas dimensiones explica en parte por qué el participante 11 adopta el sistema instalado en su casa, cuando globalmente la calidad de experiencia tiende a 0 , o al negativo según el foco 3 cuantitativo serial (Figura 5). El sistema se integra correctamente a sus dimensiones claves (especialmente, la modularidad y flexibilidad), y las demás dimensiones poseen menor importancia. Pero como nuestro modelo estadístico no pondera los puntajes en función del carácter clave de una dimensión, determinadas dimensiones "no claves" pueden hacer tender la trayectoria hacia el negativo, cuando la calidad de la experiencia es suficientemente buena como para desembocar en una adopción del sistema. De ahí la relevancia, en un principio, de realizar un análisis con varios focos.

\subsection{Foco cuantitativo radial $\left(n^{\circ} 2\right)$ : dar cuenta de la estructura dimensional de los momentos}

50 A la (in)estabilidad temporal de la dinámica de calidad de la experiencia vivida, el foco cuantitativo radial $\left(n^{\circ} 2\right)$ añade el ángulo situacional. La calidad no es lo único que fluctúa fuertemente con el paso del tiempo, entre valencias positivas, neutras y negativas, sino que las dimensiones involucradas también varían fuertemente. Así, ninguno de los momentos presentados en la Figura 7 posee la misma estructura dimensional. Esto significa que en la experiencia vivida esas dimensiones nunca se presentan de la misma forma entre diferentes momentos. A veces un momento es vivido únicamente alrededor de una dimensión, a veces alrededor de varias... Sin poder afirmar con certeza que ningún momento sea absolutamente igual, vemos que la estructura dimensional de los momentos sin duda alguna varía fuertemente de un momento a otro. 
Figure 7: Síntesis empírica de la dinámica longitudinal de apropiación dei sistema por el participante 15 , sobre la base dei foco cuantitativo radial $\left(n^{\circ} 2\right)$ y de diagnósticos locales extraídos dei foco cualitativo local $\left(n^{\circ} 1\right)$. FUA facilidad de utilización y de aprendizaje EE efectividad dei ahorro $\mathrm{CT}$ confort térmico $\mathrm{AC}$ acompanamiento $\mathrm{C}$ confianza MS modularidad y flexibilidad RF riqueza funcional ES estética.

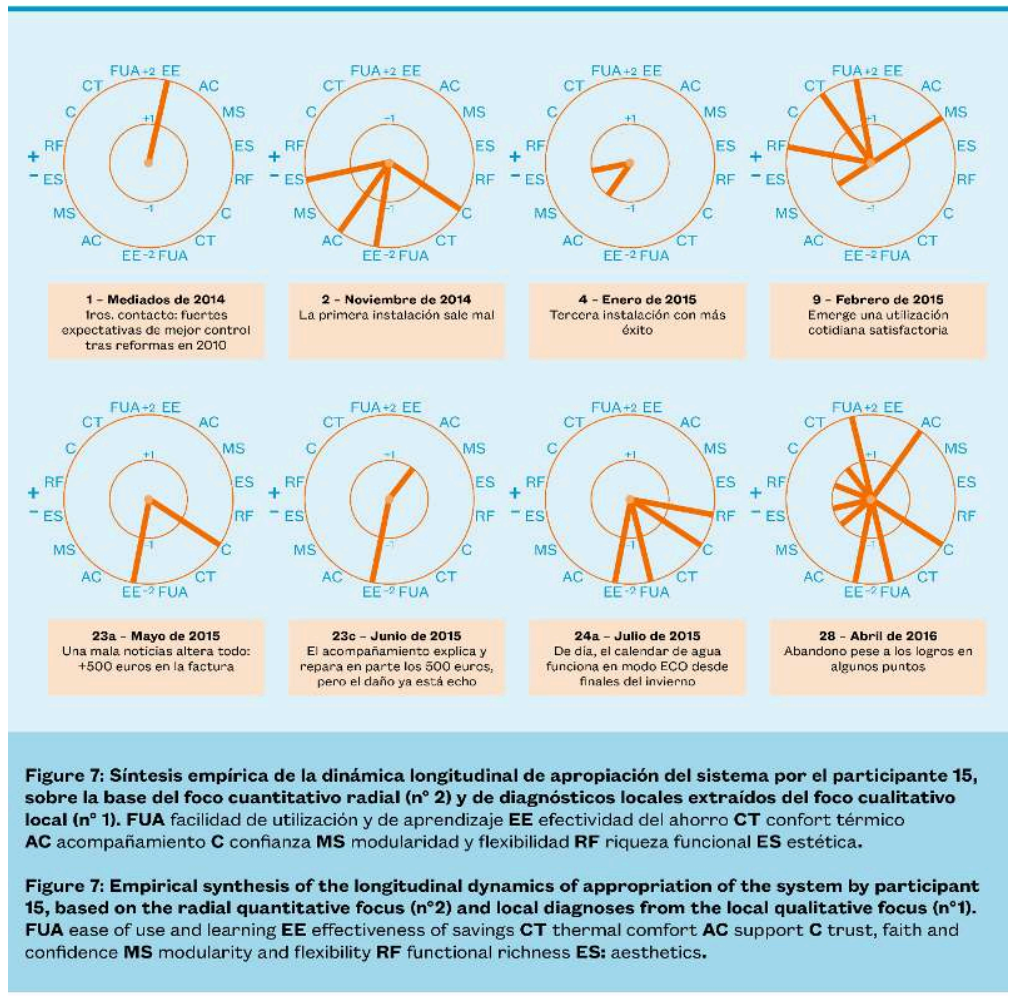

Figure 7: Síntesis empírica de la dinámica longitudinal de apropiación dei sistema por el participante 15 , sobre la base dei foco cuantitativo radial $\left(n^{\circ} 2\right)$ y de diagnósticos locales extraídos dei foco cualitativo local ( $\left.n^{\circ} 1\right)$. FUA facilidad de utilización y de aprendizaje EE efectividad dei ahorro CT confort térmico AC acompanamiento C confianza MS modularidad y flexibilidad RF riqueza funcional ES estética.

51 Este foco nos resultó útil para visualizar y hacer visualizar sintéticamente la evolución de la dinámica a lo largo de varios años, a partir de una cantidad restringida de momentos (aquí, 8). Esta selección debe ser representativa de los momentos claves (aquí, 1, 23a, 23c, 24a), pero también de los momentos que dan cuenta de la dinámica intermedia. Por ejemplo, el momento 9 antes reflejado demuestra que existió una utilización cotidiana para el participante 15 , pese a la dinámica de rechazo que emergerá más adelante. Nótese asimismo que el momento 9 no incluye vivencia alguna respecto de la dimensión clave EE: ergo, lamentablemente es poco determinante. A la inversa, los momentos 2 y 4 muestran de forma nítida que las experiencias negativas del comienzo no acarrean de inmediato un rechazo para este participante.

Este foco también permitió crear un objeto intermedio en una tableta táctil, lo cual habilita a los actores del proyecto que no tienen conocimiento de las dinámicas temporales a explorarlas libremente por sí mismos, pudiendo discutirlas con otros. Ese objeto presenta nuestros diagnósticos locales sobre los 8 momentos, además de los verbatims que dan sentido a los puntajes visibles en los radares. Además de las alternativas de concepción de 2015, este objto constituyó un modelo práctico (Theureau, 2006) final, apoyado empíricamente por el enfoque precedente. 


\section{Discusión} y sintético (y los métodos que los acompañan) para la comprensión de las dinámicas longitudinales de la actividad individual. La sección 4 demuestra ante todo que tal articulación implica un trabajo que permite: (1) cuantificar, garantizando un nexo con la experiencia vivida; (2) controlar el equilibrio entre fecundidad y peligro de "ceguera" del procesamiento cuantitativo; (3) articular varios focos de análisis-síntesis. perspectivas para futuros enfoques longitudinales de la experiencia vivida $y / o$ de la actividad individual-social. Si bien proviene de una investigación longitudinal, este marco puede ser perfectamente ajustado a contextos de investigación e intervención más restringidos en términos temporales, reduciendo la cantidad de datos recabados y afinando con mayor precisión los momentos sujetos a evaluación (véase abajo).

La sección 5 demuestra que esta articulación permite: (1) reforzar el análisis cualitativo (que sigue siendo esencial y fundamental); (2) especificar los datos cuantitativos por medio del análisis de la experiencia vivida en situación (dar carácter genérico a dinámicas en lapsos muy extendidos, limitando los riesgos de la ceguera y de la interpretación abusiva de datos); (3) visualizar con mayor facilidad las dinámicas de largo plazo y situarlas; (4) plantear nuevas preguntas, apuntalar nuevas hipótesis; (5) facilitar la activación de un razonamiento más deductivo, como complemento de la inducción/abducción que privilegia el análisis cualitativo.

cuanto a este último punto, hemos visto que si existen fuertes expectativas sobre la dimensión de Efectividad del Ahorro, entonces podemos esperar que los primeros momentos de la trayectoria no determinen la adopción/rechazo; hemos de esperar al menos dos temporadas de calefacción para ver surgir alguna forma de momento decisivo. Si no existe expectativa fuerte relativa a esta dimensión, entonces otras dimensiones pueden impactar de forma decisiva en la apropiación, pero ya desde los primeros instantes (y no después de 2 temporadas de calefacción): por ejemplo la facilidad de utilización y de aprendizaje del sistema o el acompañamiento. Lo que importa en nuestro estudio, dado el estadio incipiente de la reflexión, es más bien la posibilidad de elaborar estas formas de hipótesis y no su mera validez, puesto que ello demuestra la pertinencia de una articulación analítico-sintética para elaborar nuevos postulados y entrar en un razonamiento más deductivo. Es más, desde un punto de vista científico, sería más interesante comprobar la invalidez que confirmar esas hipótesis en su totalidad.

Este tipo de resultados y de hipótesis podría también cambiar el modo en que se piensa la recopilación de datos en los estudios sobre la apropiación. Singer y Willett (2003) consideran que a veces es posible e interesante recabar más información en los momentos críticos. Ahora bien, a tal efecto, cabe disponer de una teoría sobre qué es un momento crítico para determinado campo de conocimiento. En nuestro estudio, en el caso de fuertes expectativas en cuanto a $\mathrm{EE}$, los momentos críticos se hallan a partir de al menos dos temporadas de calefacción tras la instalación du sistema. En caso de ausencia de fuertes expectativas en torno a esa dimensión, el comienzo de la trayectoria (instalación, familiarización) puede resultar crítico y justificar recopilaciones más numerosas o más precisas. Al principio de nuestro estudio, los 39 momentos abordados por los participantes no tenían un estatus distintivo. Tales

Laboreal, Volume $16 \mathrm{~N}^{\circ} 2$ | 2020 
teorías, si bien siempre deben ser apreciadas de forma crítica, habrían permitido organizar de un modo distinto la investigación longitudinal.

Un importante límite de los modelos sintéticos presentados en este artículo es la ausencia de contribución a modo de pauta para la concepción de los sistemas y las situaciones de gestión de energía (además del tiempo de recopilación y procesamiento exigido por nuestra investigación, aunque este puede ser drásticamente reducido tratándose de intervenciones usuales en el área de ergonomía o UX research). Esto es lógico, puesto que tales modelos abarcan contenidos más genéricos, abstractos $\mathrm{y}$ desituados de la actividad humana. Aquí, el análisis cualitativo de la actividad en situación sigue siendo el enfoque más pertinente. En cambio, aplicar métodos sintéticos a una población mucho más amplia permitiría ciertamente caracterizar patrones de estabilidad y de inestabilidad (p. ej. Harbich \& Hassenzahl, 2017) en las trayectorias de apropiación de modo más consecuente; y por lo tanto guiar con mayor precisión los proyectos de concepción para pasar a escala, por ejemplo. Otro uso posible de este trabajo podría ser la concepción de herramientas informáticas más avanzadas para la caracterización o la modelización de tales trayectorias.

Nos parece, pues, que todos estos elementos allanan el camino, inclusive muy modestamente en este estadio, para un posible inicio de matematización y un diálogo en parte nuevo entre las ciencias de la complejidad y las aproximaciones a la actividad humana. No obstante, estos esfuerzos requerirían un cotejo más firme, crítico y explícito de las nociones y conceptos de cada "mundo"; cosa que, hasta ahora, sólo se hizo iterativamente en nuestro trabajo. Por ejemplo, nos cuestionamos acerca de los nexos y los contornos de las nociones de "abierto/preocupación" (extraídas del curso de acción), "dimensión clave" (extraída de nuestro trabajo intermedio, véase sección 5.2) y de "atractor" (extraída de las ciencias de la complejidad). Los "cambios profundos y duraderos en el estado de un sistema [...] se corresponden con cambios en la estructura de los atractores" (Wiese et al., 2010, p. 1027, traducción libre). La dinámica longitudinal de apropiación revelada en la sección 5.2 está fuertemente estructurada por el abierto "controlar mejor mi calefacción, saber cuánto consumo", de manera que esto parece regular la importancia de los momentos de apropiación para el participante 15 y que requeriría ponderar en alza la dimensión $\mathrm{EE}$ en el plano estadístico dentro de la serie temporal. En ese marco, ¿debería este abierto ser contemplado como un atractor de fase del binomio estructural entre el participante $15 \mathrm{y}$ su sistema de gestión de energía? ¿Como un atractor en la estructura de las dimensiones? ¿O no debería ser contemplado en absoluto como un atractor? Lo mismo ocurre respecto de la relación entre las nociones de situación (en el sentido del curso de acción), de momento (en nuestro estudio) y de vectores (en ciencias de la complejidad), por ejemplo.

Por consiguiente, el ideal de articulación analítico-sintético propuesto por Theureau $(2005,2006)$ parece absolutamente pertinente en lo que atañe a la complejidad longitudinal de la actividad humana, pero todavía necesitaría cuantiosas contribuciones para superar los límites actuales. Ese ideal apelaría a proseguir los esfuerzos de articulación entre los enfoques de la complejidad temporal: uno anclado en una fenomenología de la actividad y/o de la experiencia vivida, el otro anclado en los conceptos y modelos procedentes de los sistemas complejos. Con, en el medio, distintas hipótesis intermedias, periféricas o suplementarias, como aquellas de la actividad individual como sistema complejo, por ejemplo, o aquella de la calidad de la experiencia vivida. 
61 Por último, nos interrogamos acerca de la posible contribución del presente trabajo a otros campos que convocan el tiempo largo en ergonomía, subrayados en nuestra introducción (salud y enfermedades profesionales, gestión temporal, formación, sustentabilidad, etc.). Mardon et al. (2013) concluyen su análisis diacrónico cuantitativo de los TME recalcando que es necesario articular resultados numéricos con el análisis de la actividad, así como con entrevistas con miras retrospectivas. Nuestro estudio va plenamente en ese sentido, postulando una manera de considerar la "subjetividad" en la actividad de forma dinámica y longitudinal: entre entrevistas de puesta en situación centradas en la experiencia vivida, cuantificación situada, análisis cualitativo y síntesis cuantitativa. Asimismo, este trabajo abre perspectivas para caracterizar puntos de inflexión en las trayectorias de actividad individual (momentos claves, etc.), así como para reconsiderar la importancia que ha de otorgarse a diferentes estructuras cognitivas/emocionales (p. ej. preocupaciones, expectativas, etc.) en la evolución de la actividad a largo plazo. Resultaría interesante profundizar las discusiones cruzadas entre los distintos enfoques de los tiempo(s) largo(s) y las temporalidades múltiples de la actividad humana.

\section{BIBLIOGRAFÍA}

1Albaladejo, C., Magda, D., Geslin, P., \& Salembier, P. (2009). La mise à l'épreuve: le transfert des connaissances scientifiques en questions. Versailles: Quae.

Amalberti, R., Montmollin, M. de, \& Theureau, J. (Eds.). (1991). Modèles en analyse du travail. Liège: Mardaga.

Badiou, A. (1969). Le concept de modèle (Éd. 2007). Paris: Fayard.

Barbier, C., Cerf, M., \& Lusson, J.-M. (2015). Cours de vie d'agriculteurs allant vers l'économie en intrants: Les plaisirs associés aux changements de pratiques. Activités, 12(2). https://doi.org/ 10.4000/activites. 1081

Bationo-Tillon, A., Folcher, V., \& Rabardel, P. (2010). Les instruments transitionnels: Une proposition pour étudier la diachronie des activités narratives. Activités, 7(2). https://doi.org/ 10.4000/activites.2437

Boeing, G. (2016). Visual Analysis of Nonlinear Dynamical Systems: Chaos, Fractals, Self-Similarity and the Limits of Prediction. Systems, 4(4). https://doi.org/10.3390/systems4040037

Bourbousson, J. (2013). Cours d'action et données quantitatives: L'analyse de l'activité collective. eJRIEPS, 30. http://journals.openedition.org/ejrieps/2473

Cahour, B., Salembier, P., \& Zouinar, M. (2016). Analyzing lived experience of activity. Le Travail Humain, 79(3), 259-284.

Chizallet, M., Prost, L., \& Barcellini, F. (2019). Comprendre l'activité de conception d'agriculteurs en transition agroécologique: Vers un modèle trilogique de la conception. Psychologie Française, 64(2), 119-139. https://doi.org/10.1016/j.psfr.2019.03.001 
Daniellou, F. (2005). The French-speaking ergonomists' approach to work activity: Crossinfluences of field intervention and conceptual models. Theoretical Issues in Ergonomics Science, 6(5), 409-427. https://doi.org/10.1080/14639220500078252

Fréjus, M. (2019). Élargissement et renouvellement des questions traitées par l'ergonomie dans le domaine du développement durable: Retour sur 12 ans de travaux sur les activités domestiques et la maîtrise des consommations énergétiques. Psychologie Française, 64(2), 179-196. https:// doi.org/10.1016/j.psfr.2018.07.002

Guastello, S. J. (2017). Nonlinear dynamical systems for theory and research in ergonomics. Ergonomics, 60(2), 167-193. https://doi.org/10.1080/00140139.2016.1162851

Guibourdenche, J., Poret, C., Poizat, G., Motté, F., Haradji, Y., Salembier, P., \& Galbat, M. (2019). Sustainable Development and Energy Systems Design: Issues and Perspectives from a Francophone Activity-Centered Approach. In A. Thatcher, K. J. Zink, \& K. Fischer (Eds.), Human Factors for Sustainability (p. 329-357). Taylor \& Francis.

Haradji, Y., Guibourdenche, J., Reynaud, Q., Poizat, G., Sabouret, N., Sempé, F., Huraux, T., \& Galbat, M. (2018). De la modélisation de l'activité humaine à la modélisation pour la simulation sociale: Entre réalisme et fécondité technologique. Activités, 15(1). https://doi.org/10.4000/ activites.3106

Harbich, S., \& Hassenzahl, M. (2017). User Experience in the Work Domain: A Longitudinal Field Study. Interacting with Computers, 29(3), 306-324. https://doi.org/10.1093/iwc/iww022

Haué, J.-B. (2003). Conception d'interfaces grand public en termes de situations d'utilisation. Le cas du « Multi-Accès » (Thèse de doctorat). Université Technologique de Compiègne, France.

Hilpert, J. C., \& Marchand, G. C. (2018). Complex Systems Research in Educational Psychology: Aligning Theory and Method. Educational Psychologist, 53(3), 185-202. https://doi.org/ 10.1080/00461520.2018.1469411

Karapanos, E., Martens, J.-B., \& Hassenzahl, M. (2012). Reconstructing experiences with iScale. International Journal of Human-Computer Studies, 70(11), 849-865. https://doi.org/10.1016/ j.ijhcs.2012.06.004

Kujala, S., Roto, V., Väänänen-Vainio-Mattila, K., Karapanos, E., \& Sinnelä, A. (2011). UX Curve: A method for evaluating long-term user experience. Interacting with Computers, 23(5), 473-483. https://doi.org/10.1016/j.intcom.2011.06.005

Mardon, C., Buchmann, W., \& Volkoff, S. (2013). Un enfoque diacrónico de los TME: Uso de datos cuantitativos en una gran empresa. Laboreal, 9(1). https://doi.org/10.4000/laboreal.6085

Singer, J. D., \& Willett, J. B. (2003). Applied Longitudinal Data Analysis: Modeling Change and Event Occurrence. Oxford University Press, USA.

Thatcher, A. (2016). Longevity in a sustainable human factors and ergonomics system-of-systems. 22a SEMANA DE LA SALUD OCUPACIONAL. https://www.researchgate.net/publication/ 309606212_Longevity_in_a_sustainable_human_factors_and_ergonomics_system-of-systems

Thatcher, A., Guibourdenche, J., \& Cahour, B. (2019). Sustainable system-of-systems and francophone activity-centered approaches in ergonomics: Converging and diverging lines of dialogue. Psychologie Française, 64(2), 159-177. https://doi.org/10.1016/j.psfr.2018.07.001

Theureau, J. (1992). Le cours d'action : Analyse sémio-logique. Essai d'une anthropologie cognitive située. Peter Lang. 
Theureau, J. (2005). Les principes de la connaissance scientifique de la complexité technicoorganisationnelle selon le programme de recherche "cours d'action" en dix-sept questions. http://www.coursdaction.fr/08-nonpublies/2005-JT-T22.pdf

Theureau, J. (2006). Le cours d'action: Méthode développée. Toulouse: Octarès.

Theureau, J. (2010). Les entretiens d'autoconfrontation et de remise en situation par les traces matérielles et le programme de recherche « cours d'action ». Revue d'anthropologie des connaissances, $4(2), 287-322$.

Walker, G. H., Stanton, N. A., Salmon, P. M., Jenkins, D. P., \& Rafferty, L. (2010). Translating concepts of complexity to the field of ergonomics. Ergonomics, 53(10), 1175-1186. https:// doi.org/10.1080/00140139.2010.513453

Wiese, S. L., Vallacher, R. R., \& Strawinska, U. (2010). Dynamic Social Psychology: Complexity and Coherence in Human Experience. Social and Personality Psychology Compass, 4(11), 1018-1030. https://doi.org/10.1111/j.1751-9004.2010.00319.x

Wisner, A. (1972). Diagnosis in ergonomics: The choice of operating models in field research. Ergonomics, 15(6), 601-620. https://doi.org/10.1080/00140137208924462

Zara-Meylan, V. (2013). Modalidades de gestão do meio temporal em uma condução de processos múltiplos em situação dinâmica: Uma pesquisa em empresas de horticultura. Laboreal, 9(1), 60-65. https://doi.org/10.4000/laboreal.6188

Zara-Meylan, V. (2016). Quelles conceptions temporelles pour analyser l'activité? Une proposition issue de recherches en ergonomie dans l'horticulture. Activités, 13(1). https:// doi.org/10.4000/activites.2732

\section{NOTAS}

1. El término "momento de apropiación" se utiliza aquí para caracterizar operativamente la apropiación. Al ser esta un proceso, no pretendemos dar cuenta de su totalidad a través de una sucesión de momentos, sino que movilizamos esa sucesión de momentos como base empírica de referencia. La transformación de los conocimientos o de los modos de utilización del sistema entre dos momentos permite determinar en qué sentido un momento particular incide en la apropiación (véase Figura 5).

2. Al contrario de una estricta aplicación de una grilla de categorías extrínsecas y predefinidas.

3. Véase Singer \& Willett (2003, p. 11-12, traducción libre): “La única limitación es que, al igual que el propio tiempo, la variable temporal no puede cambiar sino de forma monótona -en otros términos, no puede cambiar de dirección. (...) Si todos son evaluados según un calendario idéntico, se dice que el conjunto de datos está estructurado en el tiempo. Si los calendarios de recopilación de datos varían de un individuo a otro, decimos que el conjunto de datos es no estructurado en el tiempo. La modelización de la evolución individual es lo suficientemente flexible como para abordar ambas posibilidades." Prosiguen: "Uno puede fácilmente construir un conjunto de datos por persona y por período, por más que cada participante tenga un calendario de recopilación de datos único. (...) Una variable temporal especialmente pensada permite asimismo que los conjuntos de datos 'personas-período' se adapten a modelos de investigación en los cuales la cantidad de mediciones varía de una persona a otra." (p.23). 


\section{RESÚMENES}

Un problema recurrente en la investigación longitudinal sobre la actividad humana es la falta de articulación entre los modelos analíticos cualitativos y los modelos sintéticos cuantitativos de la evolución dinámica. Esta ausencia crea dificultades para justificar las opciones de análisis y síntesis e impide un enfoque deductivo de la dinámica longitudinal. En el marco de una investigación empírica y tecnológica longitudinal de tres años sobre la apropiación, este artículo analiza las condiciones de producción y la utilidad de un modelo sintético cuantitativo de la complejidad longitudinal de la actividad individual, junto con un método analítico cualitativo. Más que la movilización de un único modelo de datos previo, la labor de modelización supone una articulación activa entre los métodos de modelos analíticos (clásicos en el análisis de actividades) y los métodos de modelos sintéticos. Discutimos las contribuciones y los límites del presente trabajo con respecto al análisis de la actividad efectuado por la ergonomía de lengua francesa y a las ciencias de la complejidad que se movilizan más a menudo en los factores humanos.

Um problema recorrente na investigação longitudinal sobre a atividade humana é a falta de articulação entre modelos analíticos qualitativos e modelos sintéticos quantitativos de evolução dinâmica. Esta ausência cria dificuldades na justificação das escolhas de análise e síntese e impede uma abordagem dedutiva das dinâmicas longitudinais. No âmbito de uma investigação empírica e tecnológica longitudinal de 3 anos sobre apropriação, este artigo discute as condições de produção e a utilidade de um modelo sintético quantitativo da complexidade longitudinal da atividade individual, em articulação com um método analítico qualitativo. Mais do que a mobilização de um modelo único de dados prévios, o trabalho de modelação envolve uma articulação ativa entre modelos-métodos analíticos (clássicos na análise de atividade) e modelosmétodos sintéticos. Discutimos as contribuições e limites deste trabalho no que diz respeito à análise da atividade na ergonomia de língua francesa e as ciências da complexidade mais frequentemente mobilizadas em fatores humanos.

Un problème récurrent des recherches longitudinales sur l'activité humaine est leur absence d'articulation entre modèle analytique qualitatif et modèle synthétique quantitatif d'évolution dynamique. Cette absence engendre des difficultés dans la justification des choix d'analyse et de synthèse et empêche d'aboutir à une démarche déductive sur les dynamiques longitudinales. Dans le cadre d'une recherche empirique et technologique longitudinale de 3 ans sur l'appropriation, cet article discute des conditions de production et de l'utilité d'une modélisation synthétique quantitative de la complexité longitudinale de l'activité individuelle, en articulation avec une méthode analytique qualitative. Plus que la mobilisation d'un modèle seul et pré-donné, le travail de modélisation relève d'une articulation active entre méthodes-modèles analytiques (classiques en analyse d'activité) et méthodes-modèles synthétiques. Nous discutons des apports et limites de ce travail vis-à-vis de l'analyse de l'activité en ergonomie de langue française et des sciences de la complexité plus souvent mobilisées dans les facteurs humains.

A recurring problem in longitudinal research on human activity is the lack of articulation between qualitative analytical models and quantitative synthetic models of the dynamic evolution. This absence creates difficulties in justifying the choices of analysis and synthesis and prevents a deductive approach to longitudinal dynamics. Within the framework of a 3-year longitudinal empirical and technological research on appropriation, this paper discusses the conditions of production and the usefulness of a quantitative synthetic model of the longitudinal complexity of individual activity, in conjunction with a qualitative analytical method. More than 
the mobilization of a single, pre-data model, the modeling work involves an active articulation between analytical model-methods (classical in activity analysis) and synthetic model-methods. We discuss the contributions and limits of this work with respect to activity analysis in Frenchlanguage ergonomics and the complexity sciences more often mobilized in human factors.

\section{ÍNDICE}

Palabras claves: modelización, modelo sintético, actividad individual, estudio longitudinal, complejidad

Keywords: modelling, synthetic model, individual activity, longitudinal study, complexity Mots-clés: modélisation, modèle synthétique, activité individuelle, étude longitudinale, complexité

Palavras-chave: modelação, modelo sintético, atividade individual, estudo longitudinal, complexidade

\section{AUTOR}

\section{JULIEN GUIBOURDENCHE}

AKTEN e Universidade de Genebra (Suíça), 2 chemin de la petite cerdagne - 69270 - Fontainessur-Saône - França

julien.guibourdenche@akten.fr 